\title{
Uninorms and nullnorms on the set of discrete fuzzy numbers
}

\author{
Juan Vicente Riera ${ }^{1}$ Joan Torrens ${ }^{1}$ \\ ${ }^{1}$ Department of Mathematics and Computer Science, University of the Balearic Islands. Spain.
}

\begin{abstract}
In this paper a method to extend discrete uninorms and nullnorms on the finite chain $L=\{0, \ldots, n\}$, to uninorms and nullnorms defined on the set of discrete fuzzy numbers whose support is a set of consecutive natural numbers contained in $L$ is presented. Some basic properties of discrete uninorms and nullnorms are preserved by this extension method and the structure of these kinds of aggregations is maintained too. Finally, we develop an application to obtain the group consensus opinion based on the extension of discrete uninorms and nullnorms.
\end{abstract}

Keywords: Uninorm, nullnorm, discrete fuzzy number, finite chain, lattice.

\section{Introduction}

The theory of aggregation functions has been extensively developed in last decades from both, the theoretical and the applicational points of view ([2], [12]). One of the fields which is most closely related to aggregation functions is fuzzy set theory. Not only because many of the usual fuzzy connectives like t-norms, t-conorms, uninorms, copulas are special kinds of aggregation functions, but also because many times the data to be aggregated are not crisp numbers, but fuzzy numbers and even subjective qualitative information. Moreover, the use of fuzzy mathematics $[10,13]$ allows to model the subjective and qualitative nature of such information.

Qualitative information is often interpreted to take values in a finite scale like Extremely Good, Very Good, Good, Fair, Bad, Very Bad, Extremely $\mathrm{Bad}$. In these cases, a finite chain $L$ is usually considered and several researchers have developed an extensive study of aggregation functions on $L$, usually called discrete aggregation functions. For instance, t-norms and t-conorms were characterized in [19], uninorms and nullnorms in [16], weighted means in [14] and even implications functions in $[15,17]$ and [18]. It is proved in [19] that only the number of elements of the finite chain $L$ is relevant when we deal with monotonic operations on $L$, and so the finite chain used in many of the mentioned works is the most simple one $L=\{0,1, \ldots, n\}$. (see [19] and also [11, 14, 16, 23]).

Recently, another approach deals with the possibility of extending monotonic operations on $L$ to operations on the set of discrete fuzzy numbers whose support is a set of consecutive natural numbers contained in $L$. More specifically, the concept of discrete fuzzy number was introduced in [21] as a fuzzy subset of $\mathbb{R}$ with discrete support and analogous properties to a fuzzy number. It is well known that arithmetic and lattice operations between fuzzy numbers are defined using the Zadeh's extension principle (see [13]). However, in general, for discrete fuzzy numbers this method fails and some approaches have been introduced in order to avoid such a drawback $([3,4,5]$ and $[22])$. In particular, it is proved in [5] that the set, $\mathcal{A}_{1}^{L}$, of discrete fuzzy numbers whose support is a set of consecutive natural numbers contained in $L$, is a distributive lattice. Thus, it is natural to study monotonic operations defined on $\mathcal{A}_{1}^{L}$ equipped with the usual lattice order. In this way, one approach is the one already commented of extending monotonic operations defined on $L$ to monotonic operations defined on the set $\mathcal{A}_{1}^{L}$. This was done for the special case of discrete t-norms and t-conorms in [6] and for the case of discrete means in [8].

Following with this idea we want to study in this paper the possibility of extending uninorms and nullnorms on $L$ to uninorms and nullnorms defined on $\mathcal{A}_{1}^{L}$. Moreover, we will see that some properties of these aggregations are preserved under this extension. At the end, we have shown an application of these aggregation operators to get a group consensus opinion.

\section{Preliminaries}

In this section we recall some results that will be used along the papers or we give references where they can be found.

\subsection{Discrete uninorms and nullnorms}

Let $(P ; \leq)$ be a non-trivial bounded partially ordered set (poset) with 0 and 1 as minimum and maximum elements respectively.

Definition 2.1 A triangular norm (briefly t-norm) on $P$ is a binary operation $T: P \times P \rightarrow P$ which is associative, increasing in each place, commutative and such that $T(x, 1)=x$ for all $x \in P$.

Definition 2.2 $A$ triangular conorm (t-conorm for short) on $P$ is a binary operation $S: P \times P \rightarrow P$ 
which is associative, increasing in each place, commutative and such that $S(x, 0)=x$ for all $x \in P$.

Uninorms and nullnorms are generalizations of tnorms and t-conorms as follows.

Definition 2.3 A uninorm on $P$ is a two-place function $U: P \times P \rightarrow P$ which is associative, increasing in each place, commutative, and such that there exists some element $e \in P$, called neutral element, such that $U(e, x)=x$ for all $x \in P$.

It is clear that the function $U$ becomes a t-norm when $e=1$ and a t-conorm when $e=0$.

Definition 2.4 A nullnorm on $P$ is a two-place function $G: P \times P \rightarrow P$ which is associative, increasing in each place, commutative, and such that there exists some element $k \in P$, called absorbing element, such that $G(k, x)=k$ for all $x \in P$, and satisfies

$$
\begin{array}{ll}
G(0, x)=x & \text { for all } x \leq k \\
G(1, x)=x & \text { for all } x \geq k .
\end{array}
$$

In this case nullnorms with $k=0$ lead back to tnorms, while the case $k=1$ leads back to t-conorms.

An important case is when we take as poset a finite chain $L$ with $n+1$ elements. In such a framework only the number of elements is relevant (see [19]) and so it is usually considered the most simple one, that is, $L=\{0,1, \cdots, n\}$. Operations on $L$ are usually called discrete operations and they have been studied by many authors [9, 11, 14, 16, 19, 23]. In these studies the following condition, generally used as a discrete counterpart of continuity, is usually considered.

Definition 2.5 A function $f: L \rightarrow L$ is said to be smooth if it satisfies: $|f(x)-f(x-1)| \leq 1$ for all $x \in L$ with $x \geq 1$.

Definition 2.6 A binary operation $F: L^{2} \rightarrow L$ is said to be smooth when each one of its vertical and horizontal sections $(F(x,$.$) and F(., y)$, respectively) are smooth.

Smooth discrete t-norms and t-conorms were characterized in [19]. For the case of nullnorms we have the following result.

Proposition 2.7 A binary operation $G: L \times L \rightarrow$ $L$ is a nullnorm if and only if there exist $k \in L, a$ $t$-conorm $S$ on $[0, k]$ and a $t$-norm $T$ on $[k, n]$ such that for all $x, y \in L, G$ is given by

$$
G(x, y)=\left\{\begin{array}{l}
S(x, y) \text { if }(x, y) \in[0, k]^{2} \\
T(x, y) \text { if }(x, y) \in[k, n]^{2} \\
k \text { otherwise. }
\end{array}\right.
$$

Moreover, $G$ is smooth if and only if $T$ and $S$ are smooth.
There are no smooth uninorms on $L$ with neutral element $0<e<n$ (that is, different from t-norms or t-conorms). However, when the underlying tnorm and t-conorm are smooth the corresponding uninorm can be smooth except in very restricted regions like for instance the $e$-sections. This is the case of uninorms in $U_{\min }$ and $U_{\max }$.

Definition 2.8 [16] A binary operation $U$ : $L \times$ $L \rightarrow L$ is a uninorm in $U_{\min }$ with neutral element $0<e<n$ if and only if there is a t-norm $T$ on $[0, e]$ and a t-conorm $S$ on $[e, n]$ such that $U$ is given by

$$
U(x, y)=\left\{\begin{array}{l}
T(x, y) \text { if }(x, y) \in[0, e]^{2} \\
S(x, y) \text { if }(x, y) \in[e, n]^{2} \\
\min (x, y) \text { otherwise }
\end{array}\right.
$$

Definition 2.9 [16] A binary operation $U$ : $L \times$ $L \rightarrow L$ is a uninorm in $U_{\max }$ with neutral element $0<e<n$ if and only if there is a t-norm $T$ on $[0, e]$ and a t-conorm $S$ on $[e, n]$ such that $U$ is given by

$$
U(x, y)=\left\{\begin{array}{l}
T(x, y) \text { if }(x, y) \in[0, e]^{2} \\
S(x, y) \text { if }(x, y) \in[e, n]^{2} \\
\max (x, y) \text { otherwise }
\end{array}\right.
$$

In both cases, when $T, S$ are smooth, the corresponding uninorm is also smooth except in certain points $(x, e)$ or $(e, y)$ of the $e$-sections.

\subsection{Discrete fuzzy numbers}

In this section, we recall some definitions and the main results about discrete fuzzy numbers which will be used later. By a fuzzy subset of $\mathbb{R}$, we mean a function $A: \mathbb{R} \rightarrow[0,1]$. For each fuzzy subset $A$, let $A^{\alpha}=\{x \in \mathbb{R}: A(x) \geq \alpha\}$ for any $\alpha \in(0,1]$ be its $\alpha$-level set (or $\alpha$-cut). By $\operatorname{supp}(A)$, we mean the support of $A$, i.e. the set $\{x \in \mathbb{R}: A(x)>0\}$. By $A^{0}$, we mean the closure of $\operatorname{supp}(A)$.

Definition 2.10 [21] A fuzzy subset $A$ of $\mathbb{R}$ with membership mapping $A: \mathbb{R} \rightarrow[0,1]$ is called discrete fuzzy number if its support is finite, i.e., there exist $x_{1}, \ldots, x_{n} \in \mathbb{R}$ with $x_{1}<x_{2}<\ldots<x_{n}$ such that $\operatorname{supp}(A)=\left\{x_{1}, \ldots, x_{n}\right\}$, and there are natural numbers $s, t$ with $1 \leq s \leq t \leq n$ such that:

1. $A\left(x_{i}\right)=1$ for any natural number $i$ with $s \leq i \leq$ $t$ (core)

2. $A\left(x_{i}\right) \leq A\left(x_{j}\right)$ for each natural number $i, j$ with $1 \leq i \leq j \leq s$

3. $A\left(x_{i}\right) \geq A\left(x_{j}\right)$ for each natural number $i, j$ with $t \leq i \leq j \leq n$

Remark 2.11 If the fuzzy subset $A$ is a discrete fuzzy number then the support of $A$ coincides with its closure, i.e. $\operatorname{supp}(A)=A^{0}$.

From now on, we will denote the set of discrete fuzzy numbers by $D F N$ and the abbreviation $d f n$ will denote a discrete fuzzy number. 
Theorem 2.12 [22] (Representation of discrete fuzzy numbers) Let $A$ be a discrete fuzzy number. Then the following statements (1)-(4) hold:

1. $A^{\alpha}$ is a nonempty finite subset of $\mathbb{R}$, for any $\alpha \in[0,1]$

2. $A^{\alpha_{2}} \subseteq A^{\alpha_{1}}$ for any $\alpha_{1}, \alpha_{2} \in[0,1]$ with $0 \leq$ $\alpha_{1} \leq \alpha_{2} \leq 1$

3. For any $\alpha_{1}, \alpha_{2} \in[0,1]$ with $0 \leq \alpha_{1} \leq \alpha_{2} \leq 1$, if $x \in A^{\alpha_{1}}-A^{\alpha_{2}}$ we have $x<y$ for all $y \in A^{\alpha_{2}}$, or $x>y$ for all $y \in A^{\alpha_{2}}$

4. For any $\alpha_{0} \in(0,1]$, there exist some real numbers $\alpha_{0}^{\prime}$ with $0<\alpha_{0}^{\prime}<\alpha_{0}$ such that $A^{\alpha_{0}^{\prime}}=A^{\alpha_{0}}$ (i.e. $A^{\alpha}=A^{\alpha_{0}}$ for any $\alpha \in\left[\alpha_{0}^{\prime}, \alpha_{0}\right]$ ).

Theorem 2.13 [22] Conversely, if for any $\alpha \in$ $[0,1]$, there exists $A^{\alpha} \subset \mathbb{R}$ satisfying analogous conditions to the (1)-(4)of Theorem 2.12, then there exists a unique $A \in D F N$ such that its $\alpha$-cuts are exactly the sets $A^{\alpha}$ for any $\alpha \in[0,1]$.

\subsection{Maximum and minimum of discrete fuzzy numbers}

Let $A, B$ be two dfn and $A^{\alpha}=\left\{x_{1}^{\alpha}, \cdots, x_{p}^{\alpha}\right\}, B^{\alpha}=$ $\left\{y_{1}^{\alpha}, \cdots, y_{k}^{\alpha}\right\}$ their $\alpha$-cuts respectively.

In [4], for each $\alpha \in[0,1]$, we consider the following sets,

$$
\begin{aligned}
\min (A, B)^{\alpha}= & \{z \in \operatorname{supp}(A) \wedge \operatorname{supp}(B) \text { such that } \\
& \left.\min \left(x_{1}^{\alpha}, y_{1}^{\alpha}\right) \leq z \leq \min \left(x_{p}^{\alpha}, y_{k}^{\alpha}\right)\right\} \text { and } \\
\max (A, B)^{\alpha}= & \{z \in \operatorname{supp}(A) \vee \operatorname{supp}(B) \text { such that } \\
& \left.\max \left(x_{1}^{\alpha}, y_{1}^{\alpha}\right) \leq z \leq \max \left(x_{p}^{\alpha}, y_{k}^{\alpha}\right)\right\}
\end{aligned}
$$

where $\operatorname{supp}(A) \wedge \operatorname{supp}(B)=$

$$
\{z=\min (x, y) \mid x \in \operatorname{supp}(A), y \in \operatorname{supp}(B)\}
$$

and $\operatorname{supp}(A) \vee \operatorname{supp}(B)=$

$$
\{z=\max (x, y) \mid x \in \operatorname{supp}(A), y \in \operatorname{supp}(B)\}
$$

Proposition 2.14 [4] There exist two unique discrete fuzzy numbers, that we will denote by $\operatorname{MIN}(A, B)$ and $M A X(A, B)$, such that they have the sets $\min (A, B)^{\alpha}$ and $\max (A, B)^{\alpha}$ as $\alpha$-cuts respectively.

The following result holds for $\mathcal{A}_{1}^{L}$, but is not true for the set of discrete fuzzy numbers in general(see $[5])$.

Theorem 2.15 [5] The triplet $\left(\mathcal{A}_{1}^{L}, M I N, M A X\right)$ is a bounded distributive lattice where $N \in \mathcal{A}_{1}^{L}$ (the unique discrete fuzzy number whose support is the singleton $\{n\}$ ) and $O \in \mathcal{A}_{1}^{L}$ (the unique discrete fuzzy number whose support is the singleton $\{0\}$ ) are the maximum and the minimum, respectively.

Remark 2.16 [5] Using these operations, we can define a partial order on $\mathcal{A}_{1}^{L}$ in the usual way: $A \preceq B$ if and only if $M I N(A, B)=A$, or equivalently, $A \preceq B$ if and only if $M A X(A, B)=B$ for any $A, B \in \mathcal{A}_{1}^{L}$. Equivalently, we can also define the partial ordering in terms of $\alpha$-cuts:

$$
\begin{aligned}
& A \preceq B \text { if and only if } \min \left(A^{\alpha}, B^{\alpha}\right)=A^{\alpha} \\
& A \preceq B \text { if and only if } \max \left(A^{\alpha}, B^{\alpha}\right)=B^{\alpha}
\end{aligned}
$$

The next theorem proposes a method to obtain a negation function on the bounded distributive lattice $\mathcal{A}_{1}^{L}$ from the unique strong negation $n$ on the finite chain $L$.

Proposition 2.17 [7] Let us consider the strong negation $\widetilde{n}$ on the finite chain $L=\{0,1, \cdots, n\}$. The mapping

$$
\begin{aligned}
\mathcal{N}: \mathcal{A}_{1}^{L} & \longrightarrow \mathcal{A}_{1}^{L} \\
A & \mapsto \mathcal{N}(A)
\end{aligned}
$$

is a strong negation on the bounded distributive lattice $\mathcal{A}_{1}^{L}=\left(\mathcal{A}_{1}^{L}, M I N, M A X\right)$ where $\mathcal{N}(A)$ is the discrete fuzzy number such that has as support the sets $\mathcal{N}(A)^{\alpha}=\left[\widetilde{n}\left(x_{p}^{\alpha}\right), \widetilde{n}\left(x_{1}^{\alpha}\right)\right]$ for each $\alpha \in[0,1]$ (being $A^{\alpha}=\left[x_{1}^{\alpha}, x_{p}^{\alpha}\right]$ the $\alpha$-cuts of $A$ ).

\section{Aggregation functions in $\mathcal{A}_{1}^{L}$}

Let us begin with a binary discrete aggregation function $F: L \times L \rightarrow L$ in general. Let $2^{L}$ be the set of all subsets of $L$. We will denote as well by $F$, the binary operation

$$
\begin{aligned}
F: 2^{L} \times 2^{L} & \longrightarrow 2^{L} \\
(\mathrm{X}, \mathrm{Y}) & \longmapsto F(\mathrm{X}, \mathrm{Y})
\end{aligned}
$$

where $F(\mathrm{X}, \mathrm{Y})=\{F(x, y) \mid x \in \mathrm{X}, y \in \mathrm{Y}\}$.

Note that t-norms and t-conorms on $\mathcal{A}_{1}^{L}$ were already constructed from smooth t-norms and tconorms on $L$ in [6] as follows.

Theorem 3.1 [6] Let $T(S)$ be a smooth t-norm(tconorm) on $L$ and let

$$
\begin{aligned}
& \mathcal{T}(\mathcal{S}): \mathcal{A}_{1}^{L} \times \mathcal{A}_{1}^{L} \rightarrow \mathcal{A}_{1}^{L} \\
&(A, B) \longmapsto \mathcal{T}(\mathcal{S})(A, B)
\end{aligned}
$$

be the extension of t-norm(t-conorm) $T(S)$ to $\mathcal{A}_{1}^{L}$, defined as follows $\mathcal{T}(\mathcal{S})(A, B)$ is the discrete fuzzy number whose $\alpha$-cuts are the sets $\{z \in$ $T(\operatorname{supp}(A), \operatorname{supp}(B)) \mid T(S)\left(\min A^{\alpha}, \min B^{\alpha}\right) \leq$ $\left.z \leq T(S)\left(\max A^{\alpha}, \max B^{\alpha}\right)\right\}$ for each $\alpha \in[0,1]$. Then, $\mathcal{T}(\mathcal{S})$ is a t-norm (t-conorm) on the bounded set $\mathcal{A}_{1}^{L}$.

Remark 3.2 When the t-norm $T: L \times L \rightarrow L$ is smooth it reaches all values between $\min T\left(A^{\alpha}, B^{\alpha}\right.$ and $\max T\left(A^{\alpha}, B^{\alpha}\right.$ and so the $\alpha$-cuts in the theorem above can be also written as $\mathcal{T}(A, B)^{\alpha}=$

$$
\left\{z \in L \mid \min T\left(A^{\alpha}, B^{\alpha}\right) \leq z \leq \max T\left(A^{\alpha}, B^{\alpha}\right)\right\} .
$$

Note that this is no longer true when $T$ is not smooth. 
Now, we want to proceed in a similar way but from binary aggregation functions $F$ on $L$, not necessarily smooth. By the previous remark we need to modify the definition of the $\alpha$-cut sets to be used in the construction of $F(A, B)^{\alpha}$. Thus, instead of taking $z \in F(\operatorname{supp}(A), \operatorname{supp}(B))$, we need to take $z \in L$ satisfying the corresponding condition as follows ${ }^{1}$.

Definition 3.3 Given a binary aggregation function $F$ on $L$ and two discrete fuzzy numbers $A, B \in$ $\mathcal{A}_{1}^{L}$, we define for each $\alpha \in[0,1]$ the sets

$$
\begin{aligned}
C_{F, A, B}^{\alpha}= & \left\{z \in L \mid \min F\left(A^{\alpha}, B^{\alpha}\right) \leq z\right. \\
& \left.\leq \max F\left(A^{\alpha}, B^{\alpha}\right)\right\} .
\end{aligned}
$$

Note that, from the monotonicity of the binary discrete aggregation function $F$, the set $C_{F, A, B}^{\alpha}$ can be written as

$$
\begin{aligned}
C_{F, A, B}^{\alpha}= & \left\{z \in L \mid F\left(\min A^{\alpha}, \min B^{\alpha}\right) \leq z\right. \\
& \left.\leq F\left(\max A^{\alpha}, \max B^{\alpha}\right)\right\} .
\end{aligned}
$$

for each $\alpha \in[0,1]$.

Proposition 3.4 Let us consider $A, B \in \mathcal{A}_{1}^{L}$ and let $F$ be any binary aggregation function on the finite chain $L$. There exists a unique discrete fuzzy number whose $\alpha$-cuts are exactly the sets $C_{F, A, B}^{\alpha}$ (defined in Definition 3.3), that will be denoted by $\mathcal{F}(A, B)$. Moreover, $\mathcal{F}(A, B) \in \mathcal{A}_{1}^{L}$.

The previous proposition will allow us to define a binary operation $\mathcal{F}$ on $\mathcal{A}_{1}^{L}$ from any binary aggregation function $F$ defined on the finite chain $L$.

Definition 3.5 Let us consider a binary aggregation function $F$ on the finite chain $L$. The binary operation on $\mathcal{A}_{1}^{L}$ defined as follows

$$
\begin{aligned}
\mathcal{F}: \mathcal{A}_{1}^{L} \times \mathcal{A}_{1}^{L} & \longrightarrow \mathcal{A}_{1}^{L} \\
(A, B) & \longmapsto \mathcal{F}(A, B)
\end{aligned}
$$

will be called the extension of the discrete aggregation function $F$ to $\mathcal{A}_{1}^{L}$, being $\mathcal{F}(A, B)$ the discrete fuzzy number whose $\alpha$-cuts are the sets

$$
\left\{z \in L \mid \min F\left(A^{\alpha}, B^{\alpha}\right) \leq z \leq \max F\left(A^{\alpha}, B^{\alpha}\right)\right\}
$$

for each $\alpha \in[0,1]$.

Now we want to prove that function $\mathcal{F}$ defined above is in fact a binary aggregation function on $\mathcal{A}_{1}^{L}$.

Proposition 3.6 Let $\mathcal{F}: \mathcal{A}_{1}^{L} \times \mathcal{A}_{1}^{L} \rightarrow \mathcal{A}_{1}^{L}$ be the extension of the discrete aggregation function $F$ on $L$ to $\mathcal{A}_{1}^{L}$. Let 0 and $N$ be the minimum and the maximum of $\mathcal{A}_{1}^{L}$, respectively. Then the following properties hold

$$
\begin{aligned}
& \text { 1. } \mathcal{F} \text { is increasing in each place, } \\
& \text { 2. } \mathcal{F}(O, O)=O \\
& \text { 3. } \mathcal{F}(N, N)=N .
\end{aligned}
$$

\footnotetext{
${ }^{1}$ Of course, when $F$ is smooth both expressions will coincide again as in the case of smooth t-norms.
}

That is, $\mathcal{F}$ is a binary aggregation function on $\mathcal{A}_{1}^{L}$.

Proposition 3.7 Let $\mathcal{F}: \mathcal{A}_{1}^{L} \times \mathcal{A}_{1}^{L} \rightarrow \mathcal{A}_{1}^{L}$ be the extension of the aggregation function $F$ on $L$ to $\mathcal{A}_{1}^{L}$. Then, for any $A, B, C \in \mathcal{A}_{1}^{L}$ the following properties hold.

1. If $F$ is a commutative aggregation function then its extension $\mathcal{F}$ is commutative as well, i.e. $\mathcal{F}(A, B)=\mathcal{F}(B, A)$.

2. If $F$ is a associative aggregation function then its extension $\mathcal{F}$ is associative as well, i.e. $\mathcal{F}(\mathcal{F}(A, B), C)=\mathcal{F}(A, \mathcal{F}(B, C))$.

\subsection{Uninorms in $\mathcal{A}_{1}^{L}$}

Let us deal in this section with the special case of uninorms. As in the general case we give the following definition.

Definition 3.8 Let us consider a discrete uninorm $U$ on the finite chain $L$. The binary operation on $\mathcal{A}_{1}$ defined as follows

$$
\begin{aligned}
\mathcal{U}: \mathcal{A}_{1}^{L} \times \mathcal{A}_{1}^{L} & \longrightarrow \mathcal{A}_{1}^{L} \\
(A, B) & \longmapsto \mathcal{U}(A, B)
\end{aligned}
$$

will be called the extension of the discrete uninorm $U$ to $\mathcal{A}_{1}$, being $\mathcal{U}(A, B)$ the discrete fuzzy number whose $\alpha$-cuts are the sets

$$
\left\{z \in L \mid \min U\left(A^{\alpha}, B^{\alpha}\right) \leq z \leq \max U\left(A^{\alpha}, B^{\alpha}\right)\right\}
$$

for each $\alpha \in[0,1]$.

Theorem 3.9 Let $U$ be a discrete uninorm on $L$ with $e \in L$ as neutral element. And let

$$
\begin{aligned}
\mathcal{U}: \mathcal{A}_{1}^{L} \times \mathcal{A}_{1}^{L} & \longrightarrow \mathcal{A}_{1}^{L} \\
(A, B) & \longmapsto \mathcal{U}(A, B)
\end{aligned}
$$

be the extension of $U$ to $\mathcal{A}_{1}^{L}$, defined according to Definition 3.8. Then, $\mathcal{U}$ is a uninorm on $\mathcal{A}_{1}^{L}$ with neutral element given by the unique discrete fuzzy number $E$ whose support is the singleton $\{e\}$. Moreover, if $U$ is an idempotent uninorm then so is its extension $\mathcal{U}$.

Now, we want to study the structure of these uninorms in $\mathcal{A}_{1}^{L}$. It occurs that many of the properties of the uninorm $U$ on $L$ are preserved by our construction. To see this we begin with some results.

Lemma 3.10 Let $U$ be a uninorm on the finite chain $L$ with e as neutral element. And let $E \in \mathcal{A}_{1}^{L}$ be the discrete fuzzy number such that it has only the natural number e as support. Then,

(i) For $A \in \mathcal{A}_{1}^{L}$ we have $A \preceq E$ if and only if $\operatorname{supp}(A) \subseteq[0, e]$.

(ii) For $A \in \mathcal{A}_{1}^{L}$ we have $E \preceq A$ if and only if $\operatorname{supp}(A) \subseteq[e, n]$. 
Proposition 3.11 Let $U$ be a uninorm on the finite chain $L$. Then its extension

$$
\begin{aligned}
\mathcal{U}: \mathcal{A}_{1}^{L} \times \mathcal{A}_{1}^{L} & \longrightarrow \mathcal{A}_{1}^{L} \\
(A, B) & \longmapsto \mathcal{U}(A, B)
\end{aligned}
$$

satisfies:

$$
\begin{aligned}
& \text { 1. } \mathcal{U}(A, N)=N \text { for all } A \succeq E \text { and } \mathcal{U}(A, 0)=0 \\
& \text { for all } A \preceq E \text {. } \\
& \text { 2. } \mathcal{U}(0, N) \in\{0, N\},
\end{aligned}
$$

where $N$ and 0 denote the maximum and the minimum of the bounded distributive lattice $\mathcal{A}_{1}^{L}$ respectively and $E$ is the neutral element of $\mathcal{U}$.

Now we can see that the structure of uninorms in $U_{\min }$ and $U_{\max }$ is partially preserved.

Proposition 3.12 Let us consider a uninorm

$$
U_{\min }(x, y)=\left\{\begin{array}{l}
T(x, y) \text { if }(x, y) \in[0, e]^{2} \\
S(x, y) \text { if }(x, y) \in[e, n]^{2} \\
\min (x, y) \text { otherwise }
\end{array}\right.
$$

on $L$ with neutral element $0<e<n$. And $\mathcal{U}$ its extension according to Definition 3.8. Then,

(i) If $A, B \preceq E$ then $\mathcal{U}(A, B)=\mathcal{T}(A, B)$ where $\mathcal{T}$ is the extension on $\mathcal{A}_{1}^{[0, e]}$ of the discrete triangular norm $T$.

(ii) If $A, B \succeq E$ then $\mathcal{U}(A, B)=\mathcal{S}(A, B)$ where $\mathcal{S}$ is the extension on $\mathcal{A}_{1}^{[e, n]}$ of the discrete triangular conorm $S$.

(iii) If $A \preceq E \preceq B$ then $\mathcal{U}(A, B)=\operatorname{MIN}(A, B)=$ A.

Analogously,

Proposition 3.13 Let us consider a uninorm

$$
U_{\max }(x, y)=\left\{\begin{array}{l}
T(x, y) \text { if }(x, y) \in[0, e]^{2} \\
S(x, y) \text { if }(x, y) \in[e, n]^{2} \\
\max (x, y) \text { otherwise }
\end{array}\right.
$$

on $L$ with neutral element $0<e<n$. And $\mathcal{U}$ its extension according to Definition 3.8. Then,

(i) If $A, B \preceq E$ then $\mathcal{U}(A, B)=\mathcal{T}(A, B)$ where $\mathcal{T}$ is the extension to $\mathcal{A}_{1}^{[0, e]}$ of the discrete triangular norm $T$.

(ii) If $A, B \succeq E$ then $\mathcal{U}(A, B)=\mathcal{S}(A, B)$ where $\mathcal{S}$ is the extension to $\mathcal{A}_{1}^{[e, n]}$ of the discrete triangular conorm $S$.

(iii) If $A \preceq E \preceq B$ then $\mathcal{U}(A, B)=M A X(A, B)=$ $B$.

Remark 3.14 Note that the previous theorems do not give the complete structure of uninorms in $\mathcal{A}_{1}^{L}$ that are extensions of uninorms in $U_{\min }$ and $U_{\max }$ on L. Since the order in $\mathcal{A}_{1}^{L}$ is not total, they are elements $A \in \mathcal{A}_{1}^{L}$ not comparable with the element $E$ (from Lemma 3.10 this is the case of any discrete fuzzy number $A \in \mathcal{A}_{1}^{L}$ with support not contained neither in $[0, e]$ nor in $[e, n])$. Such elements are not included in the cases of the previous propositions and thus, only the general definition of $U(A, B)$ given in Definition 3.9 works for these elements.

\subsection{Nullnorms in $\mathcal{A}_{1}^{L}$}

Let us now deal with nullnorms.

Definition 3.15 Let us consider a discrete nullnorm $F$ on the finite chain $L$. The binary operation on $\mathcal{A}_{1}$ defined as follows

$$
\begin{aligned}
\mathcal{F}: \mathcal{A}_{1}^{L} \times \mathcal{A}_{1}^{L} & \longrightarrow \mathcal{A}_{1}^{L} \\
(A, B) & \longmapsto \mathcal{F}(A, B)
\end{aligned}
$$

will be called the extension of the discrete nullnorm $F$ to $\mathcal{A}_{1}^{L}$, being $\mathcal{F}(A, B)$ the discrete fuzzy number whose $\alpha$-cuts are the sets

$$
\left\{z \in L \mid \min F\left(A^{\alpha}, B^{\alpha}\right) \leq z \leq \max F\left(A^{\alpha}, B^{\alpha}\right)\right\}
$$

for each $\alpha \in[0,1]$.

Theorem 3.16 Let $F$ be a discrete nullnorm on $L$ with $k \in L$ as absorbing element. And let

$$
\begin{aligned}
\mathcal{F}: \mathcal{A}_{1}^{L} \times \mathcal{A}_{1}^{L} & \longrightarrow \mathcal{A}_{1}^{L} \\
(A, B) & \longmapsto \mathcal{F}(A, B)
\end{aligned}
$$

be the extension of $F$ to $\mathcal{A}_{1}^{L}$, defined according to Definition 3.15. Then, $\mathcal{F}$ is a nullnorm on $\mathcal{A}_{1}^{L}$ with absorbing element the discrete fuzzy number $K \in$ $\mathcal{A}_{1}^{L}$ whose support is given by the singleton $\{k\}$.

Similarly to the case of uninorms we can easily prove the following result showing part of the structure of the extensions of nullnorms.

Proposition 3.17 Let $F$ be a nullnorm on $L$ with absorbing element $k$ and underlying t-norm $T$ and $t$-conorm $S$. Then its extension $\mathcal{F}$ satisfies the following properties:

(i) If $A, B \preceq K$ then $\mathcal{U}(A, B)=\mathcal{S}(A, B)$ where $\mathcal{S}$ is the extension to $\mathcal{A}_{1}^{[0, e]}$ of the t-conorm $S$.

(ii) If $A, B \succeq K$ then $\mathcal{U}(A, B)=\mathcal{T}(A, B)$ where $\mathcal{T}$ is the extension to $\mathcal{A}_{1}^{[e, n]}$ of the t-norm $T$.

(iii) If $A \preceq K \preceq B$ then $\mathcal{U}(A, B)=K$.

Remark 3.18 Similarly as for the case of uninorms in $U_{\min }$ and $U_{\max }$, the previous theorems do not give the complete structure of nullnorms in $\mathcal{A}_{1}^{L}$ that are extensions of nullnorms on $L$. The elements $A \in \mathcal{A}_{1}^{L}$ that are not comparable with the element $K$ (from Lemma 3.10 this is the case of any discrete fuzzy number $A \in \mathcal{A}_{1}^{L}$ with support not contained neither in $[0, k]$ nor in $[k, n])$ are not considered in this proposition, and for them only the general definition of $F(A, B)$ given in Definition 3.15 works. 


\section{Group consensus opinion based on extension of discrete uninorms}

Consensus decision-making is a group decision making process that not only seeks the agreement of most participants, but also the resolution or mitigation of minority objections. For example, in this sense, Yager [24] considers the problem of multiagent group decision making. He describes the possible use of the uninorm aggregation operator as a way of combining individual agents' preference functions to obtain a group preference function. Moreover, he provides a method to avoid the possibility of an agent using one kind of manipulation of the preference information, forcing the other group members to change their opinions towards his own opinion. Interesting studies on this subject can be found in $[20,25]$. In order to do this, Yager suggested to use discrete weighted uninorm operators defined on an ordinal scale $S=\left\{s_{0}, \cdots, s_{n}\right\}$ with $s_{i}>s_{j}$ if $i>j$, and a binary function on $S, h_{s_{g}}$ (where $s_{g}$ denoted the neutral element of the uninorm $U$ ) called importance transformation function that penalizes an agent if he uses a strategic manipulation. Recently, other interesting work on consensus decision-making is due by Alonso et al. [1] who present an implemented web based consensus support system that is able to help, or even replace, the moderator in a consensus process where experts are allowed to provide their preferences using one of many types (fuzzy, linguistic and multi-granular linguistic) of incomplete preference relations. Thus, we see that in these decision making process is usual on the one hand, to use aggregation operators defined on a finite chain for the purpose of adding the information, and on the other hand to use concepts of the fuzzy set theory in order to model incomplete or imprecise information.

In our approach, we will propose to establish a method to obtain the group consensus opinion based on the extension of discrete uninorms on $L$ to $\mathcal{A}_{1}^{L}$.

For this reason, assume that $r$ experts assess a set of parameters $\mathbf{P}=\left\{P_{1}, \cdots, P_{s}\right\}$ of the consensus process (for example, if we consider the valuation of a business investment, the parameters $P_{i}$ can be $P_{1}=$ Benefits in the short term, $P_{2}=$ Benefits in the long term, $P_{3}=$ Suitability of the investment, etc). Suppose that the assessments corresponding to a parameter $P_{i} \in \mathbf{P}$ are expressed by $r$ discrete fuzzy numbers belonging to $\mathcal{A}_{1}^{L}$. Moreover, let us denote $O_{1}^{P_{i}}, \cdots, O_{r}^{P_{i}} \in \mathcal{A}_{1}^{L}$ the evaluations of each expert corresponding to the chosen parameter.

Assume that the experts use the Table 1 to indicate their satisfaction of each parameter $P_{i} \in \mathbf{P}$ expressed as a discrete fuzzy number on $\mathcal{A}_{1}^{L}$. In this Table, the first column indicate the expert's names, the second row indicates the questions to assess $P_{i}$, the rows from the third to the antepenultimate indicate the fuzzy evaluations made by each expert $O_{j}$ corresponding to the each parameter $P_{i}$ which we

\begin{tabular}{|c|c|c|c|c|}
\hline \multirow{2}{*}{ Expert } & \multicolumn{4}{|c|}{ Questions to asses } \\
\cline { 2 - 5 } & $P_{1}$ & $P_{2}$ & $\cdots$ & $P_{s}$ \\
\hline$O_{1}$ & $O_{1}^{P_{1}}$ & $O_{1}^{P_{2}}$ & $\cdots$ & $O_{1}^{P_{s}}$ \\
\hline$O_{2}$ & $O_{2}^{P_{1}}$ & $O_{2}^{P_{2}}$ & $\cdots$ & $O_{2}^{P_{s}}$ \\
\hline$\vdots$ & $\vdots$ & $\vdots$ & $\vdots$ & $\vdots$ \\
\hline$O_{r}$ & $O_{r}^{P_{1}}$ & $O_{r}^{P_{2}}$ & $\cdots$ & $O_{r}^{P_{s}}$ \\
\hline \hline $\mathcal{O}$ & $C\left(\mathcal{O}, P_{1}\right)$ & $C\left(\mathcal{O}, P_{2}\right)$ & $\cdots$ & $C\left(\mathcal{O}, P_{s}\right)$ \\
\hline \hline \multicolumn{4}{|c}{ Consensus Opinion: $\mathcal{U}^{\prime}\left(C\left(\mathcal{O}, P_{1}\right), \cdots, C\left(\mathcal{O}, P_{s}\right)\right)$} \\
\hline
\end{tabular}

Table 1: Consensus Opinion Sheet

have denoted by $O_{j}^{P_{i}}$. The penultimate row shows the aggregations of all fuzzy assessments made by each expert on the question $P_{i}$ which we have denoted by $C\left(\mathcal{O}, P_{i}\right)=\mathcal{U}_{i}\left(O_{1}^{P_{i}}, \cdots, O_{r}^{P_{i}}\right)$. This aggregation function $\mathcal{U}_{i}$ has been obtained by using the extension of an idempotent uninorm $U_{i}$ on $L$.

Finally, the last row indicates the aggregation of all the values $C\left(\mathcal{O}, P_{i}\right)$ with $i=1, \cdots, s$, that is, the aggregation obtained using the extension of the uninorm $U^{\prime}$ on $L$ of all the $C\left(\mathcal{O}, P_{i}\right)$, $\mathcal{U}^{\prime}\left(C\left(\mathcal{O}, P_{1}\right), \cdots, C\left(\mathcal{O}, P_{s}\right)\right)$. This aggregation function $\mathcal{U}^{\prime}$ has been obtained by using the extension of an idempotent uninorm $U^{\prime}$ on $L$.

The proposed method for the consensus opinion based on the extension of a uninorm is presented as follows:

Step 1: The board of directors constitutes the expert group $E G=\left\{O_{1}, \cdots, O_{r}\right\}$ who carry out the evaluation process of the parameters $\mathbf{P}=\left\{P_{1}, \cdots, P_{s}\right\}$.

Step 2: Choose the linguistic hedges $L$ which are used to make the evaluation process.

Step 3: Each expert $O_{j}$ performs an assessment $O_{j}^{P_{i}} \in \mathcal{A}_{1}^{L}$ of all parameters $P_{i} \in \mathbf{P}$ chosen.

Step 4: For each parameter $P_{i} \in \mathbf{P}$, the company uses a uninorm $\mathcal{U}_{i}$ (obtained from the extension of an idempotent discrete uninorm $U_{i}$ defined on the finite chain $L$ ) to calculate the aggregation of all valuations $O_{1}^{P_{i}}, \cdots, O_{r}^{P_{i}}$, that is, $\mathcal{U}_{i}\left(O_{1}^{P_{i}}, \cdots, O_{r}^{P_{i}}\right)$. This aggregation will be denoted by $C\left(\mathcal{O}, P_{i}\right)$

Step 5: Finally, the board of directors (based on their experience) gets an aggregation of all the assessments $C\left(\mathcal{O}, P_{i}\right)$ made by the group of decision-makers (using a uninorm $\mathcal{U}$ obtained from the extension of an idempotent uninorm $U$ defined on the finite chain $L)$ according to the expression $\mathcal{U}\left(\mathcal{U}_{1}\left(O_{1}^{P_{1}}, \cdots, O_{r}^{P_{1}}\right), \cdots, \mathcal{U}_{s}\left(O_{1}^{P_{s}}, \cdots, O_{r}^{P_{s}}\right)\right)$ which we will denote in Table 1 by $\mathcal{U}^{\prime}\left(C\left(\mathcal{O}, P_{1}\right), \cdots, C\left(\mathcal{O}, P_{s}\right)\right)$

Remark 4.1 Note that in the previous process in order to determine the consensus opinion of a group of decision-makers we have used the extension of a 
uninorm defined on a finite chain. Moreover, if we consider in this process the extension of a nullnorm to obtain the consensus opinion, our method is a valid procedure too.

In the next examples, according to the previous method we consider the scale

$$
\mathfrak{L}=\{E B, V B, B, M B, F, M G, G, V H, E G\}
$$

where the letters refer to the linguistic terms Extremely Bad, Very Bad, Bad, More or Less Bad, Fair, More or Less Good, Good, Very Good and Extremely Good and they are listed in an increasing order:

$$
E B \prec V B \prec B \prec M B \prec F \prec M G \prec G \prec V H \prec E G
$$

It is obvious that we can consider a bijective application between this ordinal scale $\mathfrak{L}$ and the finite chain $L=\{0,1,2,3,4,5,6,7,8\}$ of natural numbers which keep the order. Furthermore, each normal convex fuzzy subset defined on the ordinal scale $\mathfrak{L}$ can be considered like a discrete fuzzy number belonging to $\mathcal{A}_{1}^{L}$, and viceversa.

Example 4.2 Suppose that a business company wants to buy a car company. For this reason, the board of directors hires three experts to determine the feasibility of this project. Assume that these three decision-makers evaluate the set of parameters $\mathbf{P}=\left\{P_{1}, P_{2}, P_{3}\right\}$ where $P_{1}=$ Benefits in the medium term, $P_{2}=$ Suitability of the investment and $P_{3}=$ Other factors. Suppose that

$$
\begin{aligned}
& O_{1}^{P_{1}}=\{0.6 / 2,1 / 3,0.8 / 4,0.7 / 5\} \\
& O_{2}^{P_{1}}=\{0.3 / 3,0.6 / 4,1 / 5,0.7 / 6\} \\
& O_{3}^{P_{1}}=\{0.7 / 2,0.8 / 3,1 / 4,0.5 / 5\} \\
& O_{1}^{P_{2}}=\{0.8 / 6,0.9 / 7,1 / 8\} \\
& O_{2}^{P_{2}}=\{0.6 / 5,0.7 / 6,1 / 7,0.7 / 8\} \\
& O_{3}^{P_{2}}=\{0.5 / 4,0.7 / 5,1 / 6,0.7 / 7,0.4 / 8\} \\
& O_{1}^{P_{3}}=\{0.1 / 0,0.6 / 1,1 / 2,0.4 / 3\} \\
& O_{2}^{P_{3}}=\{0.5 / 3,0.7 / 4,1 / 5\} \\
& O_{3}^{P_{3}}=\{0.6 / 2,0.7 / 3,1 / 4,0.8 / 5\}
\end{aligned}
$$

represent the assessments of each decision-maker corresponding to the chosen parameter $P_{i}$. Suppose that the company uses the extension of this idempotent discrete uninorm

$$
U(x, y)=\left\{\begin{array}{l}
\min (x, y) \text { if } y \leq 8-x \\
\max (x, y) \text { otherwise }
\end{array}\right.
$$

defined on the finite chain $L=\{0,1,2,3,4,5,6,7,8\}$ to obtain the consensus opinion $C\left(\mathcal{O}, P_{i}\right)$ to each parameter $P_{i}$. Finally, suppose that the business company uses the extension of this discrete idempotent uninorm

$$
U(x, y)=\left\{\begin{array}{l}
\max (x, y) \text { if }(x, y) \in[4,8]^{2} \\
\min (x, y) \text { otherwise }
\end{array}\right.
$$

defined on the finite chain $L=\{0,1,2,3,4,5,6,7,8\}$ to get the consensus opinion corresponding to the project viability. In these conditions, the evaluations of each parameter are:

$$
\begin{aligned}
& \mathcal{U}_{1}\left(O_{1}^{P_{1}}, O_{2}^{P_{1}}, O_{3}^{P_{1}}\right)=\{0.7 / 2,1 / 3,0.8 / 4,0.8 / 5,0.7 / 6\} \\
& \mathcal{U}_{2}\left(O_{1}^{P_{2}}, O_{2}^{P_{2}}, O_{3}^{P_{2}}\right)=\{0.7 / 6,0.9 / 7,1 / 8\} \\
& \mathcal{U}_{3}\left(O_{1}^{P_{3}}, O_{2}^{P_{3}}, O_{3}^{P_{3}}\right)=\{0.1 / 0,0.6 / 1,1 / 2,0.4 / 3\}
\end{aligned}
$$

Then, the fuzzy assessment of the Business Company to buy the car company is due by this discrete fuzzy number:

$$
\begin{gathered}
\mathcal{U}\left(C\left(\mathcal{O}, P_{1}\right), C\left(\mathcal{O}, P_{2}\right), C\left(\mathcal{O}, P_{3}\right)\right)= \\
\{0.1 / 0,0.6 / 1,1 / 2,0.4 / 3\}
\end{gathered}
$$

So, from the result above, the expert group considers a bad business to invest in a car company.

Another possible approach in order to get the consensus process could be using nullnorms. Note that, from Remark 4.1 the same algorithm proposed above also works for this kind of aggregations. The following example shows the effect of such approach in the same conditions of Example 4.2.

Example 4.3 In the same conditions of Example 4.2 above, if we use the extension of the nullnorm $G(x, y)=\left\{\begin{array}{l}\min (x+y, 4) \text { if }(x, y) \in[0,4]^{2} \\ \max (4, x+y-8) \text { if }(x, y) \in[4,8]^{2} \\ 4 \text { otherwise }\end{array}\right.$ instead of the extension of the uninorm

$$
U(x, y)=\left\{\begin{array}{l}
\max (x, y) \text { if }(x, y) \in[4,8]^{2} \\
\min (x, y) \text { otherwise }
\end{array}\right.
$$

defined on the finite chain $L=\{0,1, \cdots, 8\}$ in order to compute the assessment of the business company we obtain:

$$
\mathcal{G}\left(C\left(\mathcal{O}, P_{1}\right), C\left(\mathcal{O}, P_{2}\right), C\left(\mathcal{O}, P_{3}\right)\right)=\{1 / 4\}
$$

So, from the result above, the expert group considers that the parameters chosen are not sufficient to make a decision on the viability of the investment.

This last result emphasizes the divergent views among the valuations of the aggregations of each parameter $P_{i}, C\left(\mathcal{O}, P_{i}\right)$ with $i=1,2,3$.

\section{Conclusion}

In this article we have proposed a method to extend a binary aggregation function $F$ defined on a finite chain $L$ to the set $\mathcal{A}_{1}^{L}$ (of discrete fuzzy numbers whose support is a subset of consecutive natural numbers belonging to $L$ ). Moreover, we have shown that this extension $\mathcal{F}$ is a binary aggregation function on $\mathcal{A}_{1}^{L}$ that fulfils similar properties (commutativity, associativity) to the initial aggregation function $F$. In addition, we have shown that if we consider a uninorm $U$ or a nullnorm $G$ on $L$ their 
extensions $\mathcal{U}$ and $\mathcal{G}$ on $\mathcal{A}_{1}^{L}$ satisfy the conditions to be a uninorm and nullnorm. We have also studied some properties of these extensions. Finally, we have proposed an application of these aggregation operators to obtain a group consensus opinion in a decision making problem. Also, some examples regarding the possibility of investment of a business company are given.

\section{Acknowledgment}

This work has been partially supported by the MTM2009-10962 and MTM2009-10320 project grants, both with FEDER support.

\section{References}

[1] S. Alonso , E. Herrera-Viedma F. Chiclana and F. Herrera, A web based consensus support system for group decision making problems and incomplete preferences. Information Sciences 180: 4477-4495, Elsevier, 2010.

[2] G. Beliakov, A. Pradera and T. Calvo, Aggregation Functions: A Guide for Practicioners, in the series: Studies in Fuzziness and Soft Computing, 221, Springer, Berlin, Heidelberg, 2007.

[3] J. Casasnovas and J.V. Riera, On the addition of discrete fuzzy numbers. WSEAS Transactions on Mathematics, 5(5): 549-554, WSEAS Press, 2006.

[4] J. Casasnovas, J.V. Riera, Maximum and Mininum of Discrete Fuzzy numbers. In Frontiers in Artificial Intelligence and Applications: artificial intelligence research and development, 163, pages 273-280, IOS Press, 2007.

[5] J. Casasnovas and J.V. Riera, Lattice properties of discrete fuzzy numbers under extended min and max. In Proceedings IFSA-EUSFLAT, pages 647-652, July, Lisbon (Portugal), 2009.

[6] J. Casasnovas and J.V. Riera, Extension of discrete t-norms and t-conorms to discrete fuzzy numbers, Fuzzy Sets and Systems, 167(1):65-81, Elsevier, 2011.

[7] J. Casasnovas and J.V. Riera, S-implications in the set of discrete fuzzy numbers. In Proceedings IEEE-WCCI 2010, pages 2741-2747, July, Barcelona (Spain), 2010.

[8] J. Casasnovas and J.V. Riera, Weighted means of subjective evaluations. In R. Seising, V. Sanz (editors), Soft Computing in Humanities and Social Sciences. Springer-Verlag, Berlin. Forthcoming.

[9] B. De Baets, Model implicators and their characterization. In N. Sreele, editor, proceedings of the First ICSC International Symposium on Fuzzy logic, (ICSC 1995), pages A42-A49, Academic Press, 1995.

[10] D. Dubois and H. Prade, Fundamentals of Fuzzy Sets, Kluwer, Boston, 2000.
[11] L. Godo and V. Torra, On aggregation operators for ordinal qualitative information, IEEE Transactions on Fuzzy Systems, 8: 143-154, IEEE Computational Intelligence Society, 2000.

[12] M. Grabisch, J.L. Marichal, R. Mesiar and E. Pap, Aggregation functions, in the series: Encyclopedia of Mathematics and its Applications, 127, Cambridge University Press, 2009.

[13] George J. Klir and B. Yuan, Fuzzy sets and fuzzy logic ( Theory and applications). Prentice Hall, 1995.

[14] A. Kolesarova, G. Mayor and R. Mesiar, Weighted ordinal means, Information Sciences, 177: 3822-3830, Elsevier, 2007.

[15] M. Mas, M. Monserrat, J. Torrens and E. Trillas, A survey on Fuzzy Implications Functions, IEEE Transactions on fuzzy systems, 15: 11071121, IEEE Computational Intelligence Society, 2007.

[16] M. Mas, G. Mayor and J. Torrens, t-operators and uninorms on a finite totally ordered set, International Journal of Intelligent Systems, 14: 909-922, Wiley Periodicals, 1999.

[17] M. Mas, M. Monserrat and J. Torrens, Simplications and R-implications on a finite chain, Kybernetica, 40: 3-20, ÚTIA, 2004.

[18] M. Mas, M. Monserrat and J. Torrens, On two types of discretes implications, International Journal of Approximate Resoning, 40: 262-279, 2005.

[19] G. Mayor and J. Torrens, Triangular norms on discrete settings. In E.P. Klement and R. Mesiar, editors, Logical, Algebraic, Analytic, and Probabilistic Aspects of Triangular Norms, Elsevier, Netherlands, 2005, pages 189-230.

[20] F. Mata, L. Martinez and J.C. Martínez, Penalizing manipulation strategies in Consensus processes, in: Proceedings ESTYLF08(2008), pages 485-491.

[21] W. Voxman, Canonical representations of discrete fuzzy numbers, Fuzzy Sets and Systems, 54: 457-466, Elsevier, 2001.

[22] G. Wang, C. Wu and C. Zhao, Representation and Operations of discrete fuzzy numbers. Southeast Asian Bulletin of Mathematics 28: 1003-1010, 2005.

[23] R.R. Yager, Aggregation of ordinal information, Fuzzy Optim. Decis. Making, 6: 199-219, Springer, 2007.

[24] R.R. Yager, Defending against strategic manipulation in uninorm-based multi-agent decision making, European Journal of Operational Research 141: pages 217-232, Elsevier, 2002.

[25] R.R. Yager, Using Importances in Group Preference Aggregation to Block Strategic Manipulation, in: Studies in Fuzziness and Soft Computing, Aggregation operators. Physica-Verlag Heidelberg, 2002, pages 177-191. 